\title{
INVESTMENT OPTIMIZATION IN DISTRIBUTION NETWORK BASED ON FUZZY OUTAGE PARAMETERS
}

\author{
Bruno CANIZES \\ GECAD/ISEP \\ Polytechnic - Portugal \\ bmrc@isep.ipp.pt
}

\author{
João SOARES \\ GECAD/ISEP \\ Polytechnic - Portugal \\ japs@isep.ipp.pt
}

\author{
Zita VALE \\ GECAD/ISEP \\ Polytechnic - Portugal \\ zav@isep.ipp.pt
}

\author{
Hussein KHODR \\ GECAD/ISEP \\ Polytechnic - Portugal \\ hmk@isep.ipp.pt
}

\begin{abstract}
This paper presents a methodology that aims to increase the probability of delivering power to any load point of the electrical distribution system by identifying new investments in distribution components.

The methodology is based on statistical failure and repair data of the distribution power system components and it uses fuzzy-probabilistic modelling for system component outage parameters. Fuzzy membership functions of system component outage parameters are obtained by statistical records. A mixed integer nonlinear optimization technique is developed to identify adequate investments in distribution networks components that allow increasing the availability level for any customer in the distribution system at minimum cost for the system operator.

To illustrate the application of the proposed methodology, the paper includes a case study that considers a real distribution network.
\end{abstract}

\section{INTRODUCTION}

Distribution networks are usually meshed in design but they are often radially operated, in order not to increase the operation and protection complexity and costs.

Networks may be reconfigured with manual or automatic switching operations. Reconfiguration actions can be conducted with diverse goals, such as supplying loads at the minimum cost, increasing system security and reliability and enhancing power quality [1].

The reliability of distribution power systems is the ability to deliver electricity to all load points within acceptable levels of quality in the desired amount and the aim is to do it at the minimum cost. These are obviously conflicting goals, since to increase the quality and quantity of the energy delivered to customers necessarily increases the investment costs as well as the operation costs. The task of planners and operators is to find the adequate balance, taking into account the uncertainties of future conditions.

Maximizing reliability in distribution power systems implies minimizing the unaserved energy as well as load curtailment, avoiding an important monetary loss due to undelivered energy, economy damage and inconvenience to the distribution power systems users.

The reliability criteria used to support decision making can be deterministic or probabilistic. In both cases, it is necessary to make use of a consistent database and to undertake an exhaustive statistical analysis of all the available information, such as failure rates $(\lambda)$ and average repair times $(r)$ of each distribution system component. These components include overhead lines, cables, transformers, generators, switches, etc. Failure frequencies or probabilities of distribution overhead lines can be related to weather conditions and can also be impacted by the environment (such as tree falling or animal activities) and operational conditions (such as load levels) [2, 3]. Thus, a fuzzy approach allows obtaining adequate models to catch the fuzziness of component outage parameters.

This paper presents a technique that aims to increase the probability of delivering power to any load point of the electrical distribution system by identifying new investments in distribution components. These investments aim to achieve failure rates and repair time reduction. Repair time reduction can be obtained by increasing operation personnel, upgrading the automation system, upgrading the communication system, etc. The applicability of the proposed methodology is restricted to radially operated distribution systems.

A Mixed Integer Non-Linear Programming (MINLP) method is developed to identify the distribution network components for which investments allow increasing the availability level for any costumer in the distribution system (keep the light $\mathrm{ON}$ ) at minimum cost for system operator.

This paper is organized as follows: Section "Methodology" depicts an explanation of the proposed methodology. Section "Case Study" presents a case study based on real $30 \mathrm{kV}$ distribution network with one substation, 180 buses and 90 load points and a brief analysis of the obtained results. Finally, the last section presents the most relevant conclusions.

\section{METHODOLOGY}

A methodology to minimize the investment cost in distribution networks in order to increase the availability level for any customer is presented in this paper.

Figure 1 presents the basic idea of the proposed method.

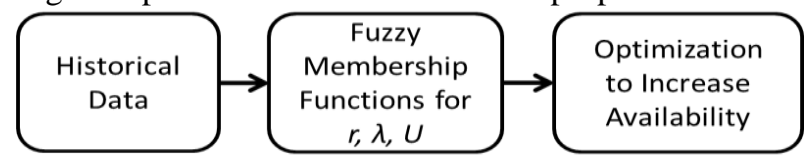

Figure 1: Diagram of the proposed methodology 
- Outage parameters data base

With all available information like repair times, number of repairs, number of failures, number of reparable failures, and time periods it is possible to develop a consistent data base and to undertake an exhaustive statistical analysis.

- Fuzzy set functions for $\bar{r}, \lambda$ and $U$

It is extremely difficult to distinguish precisely the effects of the weather conditions, and environment and operational conditions on the outage data of individual components using a probability model since there are no or little statistics available. The failure frequency or probability of distribution power systems are directly impacted by these conditions. It is also very common that a large number of utilities do not have sufficient statistical records of outage parameters.

Membership function for repair time $-\bar{r}$

A direct average of repair times in different outage events can be calculated by equation (1). Where $\bar{r}$ is the point estimate of repair time, $r_{i}$ is the ith repair time, and $n$ is the number of repair times in the outage data [4-6].

$\bar{r}=\frac{1}{n} \sum_{i=1}^{n} r_{i}$

A $t$-distribution can be used to estimate the confidence interval of the expected repair time, so [5-7]:

$r_{2}=\bar{r}-t \alpha / 2(n-1) \frac{s}{\sqrt{n}}$

$r_{3}=\bar{r}+t \alpha / 2(n-1) \frac{s}{\sqrt{n}}$

$r_{2} \leq \mu \leq r_{3}$

The real expected repair time $(\mu)$ is located between the lower $\left(r_{2}\right)$ and higher bound $\left(r_{3}\right)$ as it can be seen in equation (4). A triangle membership function for repair time $r$ (in hours per failure) can be easily created using the point and interval estimates.

Failure frequency - $f /$ failure rate $-\lambda$

Failure frequency is estimated as average failures per year over a time period for individual components.

$f=\frac{R_{f}}{T}$

where $R_{f}$ is the number of component repairable failures in time period $T$ (in years) [4-6].

Developing a theoretical accurate method for failure frequency estimation is not easy. Fortunately, statistics theory can be used to estimate the confidence interval of the failure rate, which can be approximately considered as an interval estimate of failure frequency, as they are numerically close in most cases.

In the failure data processing a relationship between Chi-Square distribution $\left(\chi^{2}\right)$ and the Poisson distribution can be considered. For a given significant level $\alpha$ the failure rate $\lambda$ falls into the random confidence interval (6) with the probability of $1-\alpha$ [57]:

$\frac{\chi_{1-\alpha / 2}^{2}(2 \cdot F)}{2 \cdot T} \leq \lambda \leq \frac{\chi_{\alpha / 2}^{2}(2 \cdot F)}{2 \cdot T}$

where $F$ is the statistical number of failures within the time $\mathrm{T}$ in data samples.

The two bounds of the failure rate can be estimated by equation (7) $[6,7]$ :

$\lambda_{2}=\frac{\chi_{1-\alpha / 2}^{2}(2 \cdot F)}{2 \cdot T}$

$\lambda_{3}=\frac{\chi_{\alpha / 2}^{2}(2 \cdot F)}{2 \cdot T}$

$\lambda_{2} \leq \lambda \leq \lambda_{3}$

A triangle membership function of failure rate can be easily created using the point and interval estimates of the failure rate.

Unavailability - U

Failure frequency (failure rate) and repair time are used to determine the unavailability $(U)$ or forced outage rate [5-7]:

$U \cong \lambda \times r$

The interval calculation rules for a given membership function grade are used to obtain the unavailability membership function.

\section{Desfuzzification}

The use of the centroid of the function obtained by the gravity technique is an appropriated approach and it is very similar to the weighted mean used in the probability theory. This technique finds the balance point calculating the weighted mean of the membership functions, and it is formulated as $[6,7]$ :

$Q_{i}=\frac{\sum_{j \epsilon K} Q_{j} \times \delta\left(Q_{j}\right)}{\sum_{j \epsilon K} \delta\left(Q_{j}\right)}$

where $Q_{j}$ is the domain point value in the membership function, $\delta\left(Q_{j}\right)$ is the membership grade for the domain 
point and $K$ is the set of domain points to be considered. The point representing the gravity center of the membership function of ith component is $Q_{i}$.

- Optimization to increase the availability

Once obtained the fuzzy membership functions for repair time, failure rate and unavailability of each system component and also the location (bus) of each load point, we can obtain the supply paths for each one of these load points. With these paths we are able to apply the MINLP model (Figure 2). The load point path corresponds to the set of components (lines/cables) in radial based operation that can feed the load point from the substation.

The MINLP methodology is developed to identify the distribution network components for which investments allow increasing the availability level for any customer in the distribution system. The goal of these investments is to achieve a reduction in repair times of system components. The repair time reduction can be achieved by increasing the operation personal, upgrading the automation system, communication system, etc. Thus, an optimization model (12-15) can be formulated.

\section{Mixed Integer Non-Linear Programming Model}

Objective function

$\operatorname{MIN} \sum_{i=1}^{N E} \sum_{m=1}^{N m} C_{i m} \cdot X_{i m}$

\section{Constraints}

$\Delta U_{j} \leq \sum_{k=1}^{N C} \sum_{m=1}^{N m} \lambda_{k} \cdot \Delta r_{k m} \cdot X_{k m} \quad k \epsilon i$

$\Delta r_{i m}=A c t_{i m}$

$\sum_{i=1}^{N E} \sum_{m=1}^{N m} X_{i m} \leq 1$

where:

$i$ is the $i$ th system component; $N e$ is the total number of system components; $m$ is the action to take for repair time reduction; $\mathrm{Nm}$ is the total number of actions for repair time reduction; $C_{i m}$ the cost in monetary units (m.u.) for the ith component with action $m$; $X_{i m}$ the decision variable; $j$ is the $j$ th component linked to the load (equal to number of loads), $\Delta U_{j}$, the unavailability variation in $j$ th component; $k$ the component that belongs to the supply path of $j t h$ component; $N C$ the total number of components that make the jth component supply path; $\lambda_{k}$ the failure rate of $k$ th component; $\Delta r_{k m}$ the repair time variation for $k t h$ component in action $\mathrm{m} ; \Delta r_{i m}$ the repair time variation for ith component in action $m$; $A c t_{i m}$ the constant value of repair time variation for ith component in action $m$.

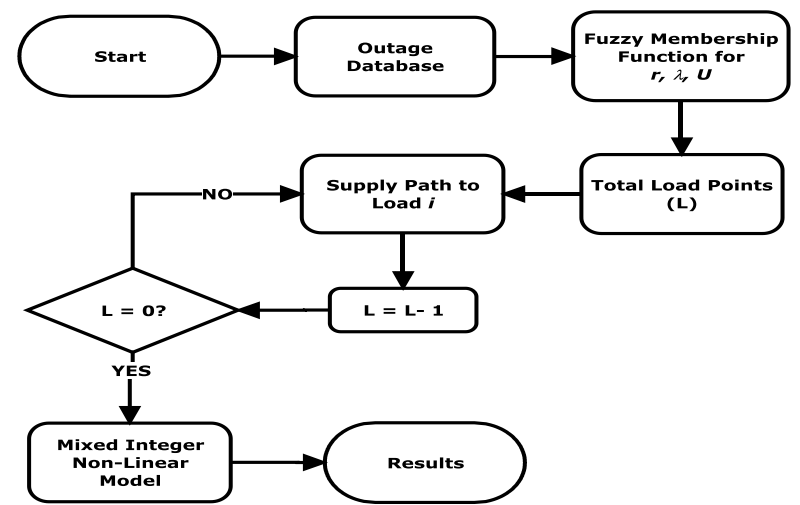

Figure 2: Methodology flowchart

It is important to note in (13) that the components which are considered to obtain the desired unavailability variation are the components that belong to the supply path of each load point (components in series). For better understanding let us consider that the system operator wants to reduce the unavailability in component 6-8 of the network presented in Figure 3 (increase the availability level for load in bus 8 ). The supply path for this load is presented in Table 1.

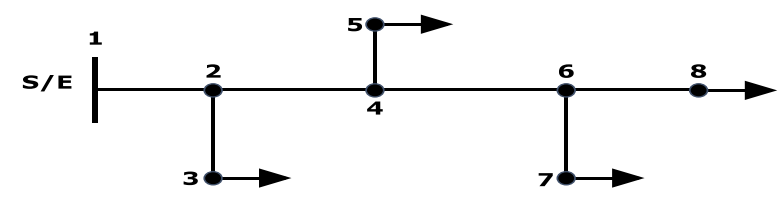

Figure 3: Eight buses distribution network

Table 1: Load supply path

\begin{tabular}{cc}
\hline \hline \multicolumn{2}{c}{ Components (Lines/Cables) } \\
\hline Bus Out & Bus In \\
\hline 1 & 2 \\
2 & 4 \\
4 & 6 \\
6 & 8 \\
\hline \hline
\end{tabular}

\section{CASE STUDY}

A $30 \mathrm{kV}$ distribution network is used to illustrate the application of the proposed methodology. This distribution network has one substation, 180 buses, 90 load points and 163 overhead lines and 16 cables. In this case study only the lines and cables were considered for the analyses. The System Operator (SO) can apply three actions in order to reduce the repair time - Increase the Operation Personal (IOP), Automation System Upgrade (ASU), Communications Upgrade (CUp) - each one with a cost and a repair time reduction for each system component. In this case study the authors considers 
which are some components to with the SO cannot apply any action (lines 5-7, 11-13, 31-34, 95-96 and cable 1-2). The SO goal is to achieve a $25 \%$ unavailability reduction of supply in each load point. All necessary data of this network as well as the respective diagram can be found in [8].

To determine the supply path of each load point an algorithm was developed in MATLAB language while MINLP model has been developed in GAMS using the CPLEX and CONOPT solvers. The case study has used a PC compatible with one processor Intel Xeon W3520 $2.67 \mathrm{GHz}$ with 4 cores, $3 \mathrm{~GB}$ of Random-AccessMemory (RAM) and Windows 7 Professional 32-Bit Operating System.

Table 2 shows the identified components by the MINLP model for which investments allows increasing the availability (with $25 \%$ desire unavailability reduction) level for any load point as well as the action to be taken.

Table 2: Identified components to apply the investment

\begin{tabular}{ccc}
\hline \hline $\begin{array}{c}\text { Components (Lines/Cables) } \\
\text { Bus Out }\end{array}$ & Action \\
\hline 2 & 3 & ASU \\
3 & 4 & CUp \\
3 & 5 & IOP \\
34 & 36 & IOP \\
100 & 102 & CUp \\
\hline \hline
\end{tabular}

The investments in these 5 components considering the action given by MINLP model have a cost of 1702 m.u. As it can be seen with investments in only 5 components the SO can achieve the $25 \%$ unavailability reduction in the supply level of each load point (90 load points).

The computational time for the MINLP model was 0.32 seconds.

Table 3 presents the repair time after the actions taken.

Table 3: Repair time after investment in selected components

\begin{tabular}{|c|c|c|c|c|}
\hline \multicolumn{2}{|c|}{$\begin{array}{c}\text { Components } \\
\text { (Lines/Cables) }\end{array}$} & \multirow{2}{*}{$\begin{array}{l}\text { Initial } \\
\text { Repair Time } \\
\text { (hours) }\end{array}$} & \multirow{2}{*}{$\begin{array}{c}\text { Repair } \\
\text { Time } \\
\text { Reduction } \\
\text { (hours) }\end{array}$} & \multirow{2}{*}{$\begin{array}{c}\text { Final Repair } \\
\text { Time } \\
\text { (hours) }\end{array}$} \\
\hline $\begin{array}{l}\text { Bus } \\
\text { Out }\end{array}$ & $\begin{array}{c}\text { Bus } \\
\text { In }\end{array}$ & & & \\
\hline 2 & 3 & 11.70 & 8.20 & 3.50 \\
\hline 3 & 4 & 9.20 & 4.50 & 4.70 \\
\hline 3 & 5 & 11.90 & 2.30 & 9.60 \\
\hline 34 & 36 & 12.10 & 2.40 & 9.70 \\
\hline 100 & 102 & 9.40 & 4.70 & 4.70 \\
\hline
\end{tabular}

\section{CONCLUSIONS}

This paper proposes a methodology that aims to increase the probability of delivering power to any load point of the electrical distribution system by identifying new investments in distribution components.

An optimization model based on MINLP was developed in order to obtain the system components in which the system operator must invest at minimum cost as well as the actions to be taken to obtain the desire unavailability reduction of supply in each load point.

For the presented case study the system operator only needs to invest in five components in order to achieve the desire unavailability reduction.

This method proved to be a good basis for a network operator decision support tool, allowing to support operator's in network investment actions.

\section{ACKNOWLEDGEMENTS}

This work is supported by FEDER Funds through the "Programa Operacional Factores de Competitividade COMPETE" program and by National Funds through FCT "Fundação para a Ciência e a Tecnologia" under the projects: FCOMP-01-0124-FEDER-PEstOE/EEI/UI0760/2011; $\quad$ FCOMP-01-0124-FEDERPTDC/EEA-EEL/099832/2008 and FCOMP-01-0124FEDER-PTDC/SEN-ENR/099844/2008.

\section{REFERENCES}

[1] D. Das, "Reconfiguration of distribution system using fuzzy multi-objective approach," International Journal of Electrical Power \& Energy Systems, vol. 28, pp. 331-338, Jun 2006.

[2] R. Billinton and W. Y. Li, "A Novel Method for Incorporating Weather Effects in Composite System Adequacy Evaluation," IEEE Transactions on Power Systems, vol. 6, pp. 1154-1160, Aug 1991.

[3] IEEE, "Electric Delivery System Reliability Evaluation," IEEE Tutorial Course Textbook No. 05TP175, 2005.

[4] W. Li, Risk assessment of power systems : models, methods, and applications. Hoboken, N.J. ; [Great Britain]: Wiley, 2005.

[5] Z. Vale, B. Canizes, J. Soares, P. Oliveira, T. Sousa, M. Silva, A. Soeiro, and H. Khodr, "Hybrid Fuzzy Monte Carlo and Logic Programming Model for Distribution Network Reconfiguration in the Presence of Outages," presented at the 21st International Conference and Exhibition on Electricity Distribution (CIRED 2011), Frankfurt, Germany, 2011.

[6] B. Canizes, Z. A. Vale, J. P. Soares, and H. M. Khodr, "Fuzzy Monte Carlo Mathematical Model for Load Curtailment Minimization in Transmission Power Systems," presented at the 17th Power Systems Computation Conference (PSCC'11), Stockholm, Sweden, 2011.

[7] W. Y. Li, J. Q. Zhou, K. G. Xie, and X. F. Xiong, "Power system risk assessment using a hybrid method of fuzzy set and Monte Carlo simulation," IEEE Transactions on Power Systems, vol. 23, pp. 336-343, May 2008.

[8] GECAD. Available: http://www.gecad.isep.ipp.pt/mascem/sobre.aspx 